\title{
COMPUTATION OF SEVERAL HESSENBERG DETERMINANTS
}

\author{
FENG $\mathrm{QI}^{1,2,3}$, OMRAN KOUBA $^{4}$, AND ISSAM KADDOURA $^{5}$ \\ Dedicated to people facing and battling COVID-19
}

\begin{abstract}
In the paper, employing methods and techniques in analysis and linear algebra, the authors find a simple formula for computing an interesting Hessenberg determinant whose elements are products of binomial coefficients and falling factorials, derive explicit formulas for computing some special Hessenberg and tridiagonal determinants, and alternatively and simply recover some known results.
\end{abstract}

\section{CONTEnts}

1. Motivations 1

2. An interesting Hessenberg determinant 3

$\begin{array}{lr}3 . \quad \text { Corollaries } & 9\end{array}$

\begin{tabular}{lll}
\hline 4. & Recovery of the identities (1.2), (1.3), and (1.4) & 11
\end{tabular}

$\begin{array}{ll}\text { References } & 13\end{array}$

\section{Motivations}

In [7, p. 99], it is stated that if $\phi^{\prime \prime}(x)>0$ for $x \in \mathbb{R}$ and $\phi(0)=0$, then the function

$$
h(x)= \begin{cases}\frac{\phi(x)}{x}, & x \neq 0 \\ \phi^{\prime}(0), & x=0\end{cases}
$$

increases with respect to $x \in \mathbb{R}$. See also [15, Remark 3] and [16, Lemma 1].

We observe that this conclusion is a simple consequence of [1, Theorem 1.25] which reads that, if $\frac{g^{\prime}(x)}{f^{\prime}(x)}$ is increasing (or decreasing, respectively) on $(a, b)$, then so are the functions

$$
\frac{g(x)-g(a)}{f(x)-f(a)} \text { and } \frac{g(x)-g(b)}{f(x)-f(b)},
$$

where $g, f:[a, b] \rightarrow \mathbb{R}$ are continuous on $[a, b]$ and differentiable on $(a, b)$ and $f^{\prime}(x) \neq 0$ on $(a, b)$ for $-\infty<a<b<\infty$. More simply, we also observe that

$$
h(x)=\int_{0}^{1} \phi^{\prime}(x t) \mathrm{d} t, \quad h^{\prime}(x)=\int_{0}^{1} t \phi^{\prime \prime}(x t) \mathrm{d} t,
$$

2010 Mathematics Subject Classification. Primary 15A15; Secondary 05A10, 11B65, 11C20, 15B36, 26A06, $65 \mathrm{~F} 40$.

Key words and phrases. explicit formula; Hessenberg determinant; tridiagonal determinant; falling factorial; rising factorial; binomial coefficient; derivative; recovery.

Please cite this article as "Feng Qi, Omran Kouba, and Issam Kaddoura, Computation of several Hessenberg determinants, Mathematica Slovaca 71 (2021), in press; available online at https://doi.org/10.1515/ms-2021-? ???"

This paper was typeset using $\mathcal{A M S}_{\mathcal{M}}$-LATEX. 
and, due to $\phi^{\prime \prime}(x)>0$ for $x \in \mathbb{R}, h(x)$ is increasing with $x \in \mathbb{R}$.

In [4, Lemma 1], it was obtained that, if $\phi(x)$ is infinitely differentiable on $\mathbb{R}$ and $\phi(0)=0$, then the function $h(x)$ defined by (1.1) satisfies

$$
h^{(n)}(x)= \begin{cases}\frac{1}{x^{n+1}} \sum_{k=0}^{n}\left(\begin{array}{l}
n \\
k
\end{array}\right)(-1)^{k} k ! x^{n-k} \phi^{(n-k)}(x), & x \neq 0 \\
\frac{1}{n+1} \phi^{(n+1)}(0), & x=0\end{cases}
$$

and

$$
\frac{\mathrm{d}}{\mathrm{d} x} \sum_{k=0}^{n}\left(\begin{array}{l}
n \\
k
\end{array}\right)(-1)^{k} k ! x^{n-k} \phi^{(n-k)}(x)=x^{n} \phi^{(n+1)}(x) .
$$

In [5. Theorem 2.1], it was proved that, if $\phi(x)$ is infinitely differentiable on $\mathbb{R}$ and $\phi^{(k-1)}(0)=0$ for $1 \leq k \leq m$, then the function

$$
h_{m}(x)= \begin{cases}\frac{\phi(x)}{x^{m}}, & x \neq 0 \\ \frac{\phi^{(m)}(0)}{m !}, & x=0\end{cases}
$$

satisfies

$$
\begin{gathered}
h_{m}^{(n)}(x)= \begin{cases}\frac{1}{x^{n+m}} \sum_{k=0}^{n}\left(\begin{array}{l}
n \\
k
\end{array}\right)(-1)^{k}(m)_{k} x^{n-k} \phi^{(n-k)}(x), & x \neq 0 ; \\
\frac{n !}{(n+m) !} \phi^{(m+n)}(0), & x=0,\end{cases} \\
\frac{\mathrm{d}^{m}}{\mathrm{~d} x^{m}} \sum_{k=0}^{n}\left(\begin{array}{l}
n \\
k
\end{array}\right)(-1)^{k}(m)_{k} x^{n-k} \phi^{(n-k)}(x)=x^{n} \phi^{(n+m)}(x),
\end{gathered}
$$

and that

$$
\phi^{(n+m)}(x) \gtreqless 0 \text { implies } h_{m}^{(n)}(x) \gtreqless 0
$$

for all $n \in \mathbb{N}$, where the quantity

$$
(z)_{n}=\prod_{\ell=0}^{n-1}(z+\ell)= \begin{cases}z(z+1) \cdots(z+n-1), & n \geq 1 \\ 1, & n=0\end{cases}
$$

is called the rising factorial of $z \in \mathbb{C}$, while

$$
\langle z\rangle_{n}=\prod_{\ell=0}^{n-1}(z-\ell)= \begin{cases}z(z-1) \cdots(z-n+1), & n \geq 1 \\ 1, & n=0\end{cases}
$$

is called the falling factorial of $z \in \mathbb{C}$. See the papers [31, 33] and closely related references therein.

A matrix $H=\left(h_{i, j}\right)_{n \times n}$ is called a lower or upper Hessenberg matrix if $h_{i, j}=0$ for all pairs $(i, j)$ such that $i+1<j$ or $h_{i, j}=0$ for all pairs $(i, j)$ such that $j+1<i$. The determinant of a lower or upper Hessenberg matrix is called a Hessenberg determinant. For more information, see the papers [11, 19, 23, 35, 39] and closely related references therein.

Let $p(t)$ and $q(t) \neq 0$ are $n$-times differentiable functions. Exercise 5 in [3] p. 40] reads that

$$
\frac{\mathrm{d}^{n}}{\mathrm{~d} t^{n}}\left[\frac{p(t)}{q(t)}\right]=\frac{(-1)^{n}}{q^{n+1}(t)}\left|W_{(n+1) \times(n+1)}(t)\right|,
$$

where

$$
\left|W_{(n+1) \times(n+1)}(t)\right|=\left|P_{(n+1) \times 1}(t) \quad Q_{(n+1) \times n}(t)\right|
$$


is an $(n+1) \times(n+1)$ Hessenberg determinant, the elements of the $(n+1) \times 1$ matrix $P_{(n+1) \times 1}(t)$ are $P_{k, 1}(t)=p^{(k-1)}(t)$ for $1 \leq k \leq n+1$, and the elements of the $(n+1) \times n$ matrix $Q_{(n+1) \times n}(t)$ are

$$
Q_{i j}(t)= \begin{cases}\left(\begin{array}{l}
i-1 \\
j-1
\end{array}\right) q^{(i-j)}(t), & i-j \geq 0 \\
0, & i-j<0\end{cases}
$$

for $1 \leq i \leq n+1$ and $1 \leq j \leq n$. The formula 1.5 has been employed in the papers [9, 10, 11, 12, 13, 14, 18, 19, 20, 21, 22, 23, 24, 25, 26, 28, 29, 30, 32, 34, 35, 36, 37, 38, 39, 43. and closely related references.

The formula 1.2 for $x \neq 0$ was established by the Leibniz theorem for differentiation of the product of $\frac{1}{x^{m}}$ and $\phi(x)$. Motivated by the formula (1.5), we can alternatively compute the $n$th derivative $h_{m}^{(n)}(x)$ by regarding $h_{m}(x)$ as a ratio between $\phi(x)$ and $x^{m}$. In this paper, starting from this idea, we will find a simple formula for computing an interesting Hessenberg determinant whose elements are products of binomial coefficients and falling factorials, derive explicit formulas for computing some special Hessenberg and tridiagonal determinants, and alternatively and simply recover main results in the paper [5].

\section{An interesting Hessenberg Determinant}

By virtue of the formula (1.5) (regarding $h_{\alpha}(x)$ as a ratio between $\phi(x)$ and $x^{\alpha}$ ) and the Leibniz theorem for differentiation of a product (regarding $h_{\alpha}(x)$ as the product of $\phi(x)$ and $x^{-\alpha}$ ) for $\alpha \in \mathbb{R}$, we can establish different expressions for the $n$th derivative of $h_{\alpha}(x)$. By comparing these two different expressions and using other techniques, we can discover the following theorem.

Theorem 2.1. For $m \in\{0\} \cup \mathbb{N}, \alpha \in \mathbb{C}$, and $\ell \in \mathbb{N}$, we have

$$
\begin{gathered}
\left|\begin{array}{cccccc|}
\left(\begin{array}{c}
m+1 \\
1
\end{array}\right)\langle\alpha\rangle_{1} & \left(\begin{array}{c}
m+1 \\
0
\end{array}\right)\langle\alpha\rangle_{0} & 0 & \cdots & 0 & 0 \\
\left(\begin{array}{c}
m+2 \\
2
\end{array}\right)\langle\alpha\rangle_{2} & \left(\begin{array}{c}
m+2 \\
1
\end{array}\right)\langle\alpha\rangle_{1} & \left(\begin{array}{c}
m+2 \\
0
\end{array}\right)\langle\alpha\rangle_{0} & \cdots & 0 & 0 \\
\left(\begin{array}{c}
m+3 \\
3
\end{array}\right)\langle\alpha\rangle_{3} & \left(\begin{array}{c}
m+3 \\
2
\end{array}\right)\langle\alpha\rangle_{2} & \left(\begin{array}{c}
m+3 \\
1
\end{array}\right)\langle\alpha\rangle_{1} & \cdots & 0 & 0 \\
\vdots & \vdots & \vdots & \ddots & \vdots & \vdots \\
\left(\begin{array}{c}
m+\ell-2 \\
\ell-2
\end{array}\right)\langle\alpha\rangle_{\ell-2} & \left(\begin{array}{c}
m+\ell-2 \\
\ell-3
\end{array}\right)\langle\alpha\rangle_{\ell-3} & \left(\begin{array}{c}
m+\ell-2 \\
\ell-4
\end{array}\right)\langle\alpha\rangle_{\ell-4} & \cdots & \left(\begin{array}{c}
m+\ell-2 \\
0
\end{array}\right)\langle\alpha\rangle_{0} & 0 \\
\left(\begin{array}{c}
m+\ell-1 \\
\ell-1
\end{array}\right)\langle\alpha\rangle_{\ell-1} & \left(\begin{array}{c}
m+\ell-1 \\
\ell-2
\end{array}\right)\langle\alpha\rangle_{\ell-2} & \left(\begin{array}{c}
m+\ell-1 \\
\ell-3
\end{array}\right)\langle\alpha\rangle_{\ell-3} & \cdots & \left(\begin{array}{c}
m+\ell-1 \\
1
\end{array}\right)\langle\alpha\rangle_{1} & \left(\begin{array}{c}
m+\ell-1 \\
0
\end{array}\right)\langle\alpha\rangle_{0} \\
\left(\begin{array}{c}
m+\ell \\
\ell
\end{array}\right)\langle\alpha\rangle_{\ell} & \left(\begin{array}{c}
m+\ell \\
\ell-1
\end{array}\right)\langle\alpha\rangle_{\ell-1} & \left(\begin{array}{c}
m+\ell \\
\ell-2
\end{array}\right)\langle\alpha\rangle_{\ell-2} & \cdots & \left(\begin{array}{c}
m+\ell \\
2
\end{array}\right)\langle\alpha\rangle_{2} & \left(\begin{array}{c}
m+\ell \\
1
\end{array}\right)\langle\alpha\rangle_{1}
\end{array}\right| \\
=\left|\left(\begin{array}{c}
m+i \\
i-j+1
\end{array}\right)\langle\alpha\rangle_{i-j+1}\right|_{1 \leq i, j \leq \ell}
\end{gathered}
$$

First proof. Let $\phi(x)$ be infinitely differentiable on $\mathbb{R}$ and $\alpha \in \mathbb{R}$. Then, by virtue of the Leibniz theorem for differentiation of a product of two functions, we have

$$
\begin{gathered}
{\left[h_{\alpha}(x)\right]^{(n)}=\left[\frac{\phi(x)}{x^{\alpha}}\right]^{(n)}=\sum_{k=0}^{n}\left(\begin{array}{l}
n \\
k
\end{array}\right)\left(\frac{1}{x^{\alpha}}\right)^{(k)} \phi^{(n-k)}(x)=\sum_{k=0}^{n}\left(\begin{array}{l}
n \\
k
\end{array}\right) \frac{\langle-\alpha\rangle_{k}}{x^{\alpha+k}} \phi^{(n-k)}(x)} \\
=\frac{1}{x^{\alpha+n}} \sum_{k=0}^{n}\left(\begin{array}{l}
n \\
k
\end{array}\right)(-1)^{k}(\alpha)_{k} x^{n-k} \phi^{(n-k)}(x) \\
=\frac{1}{x^{\alpha+n}} \sum_{k=0}^{n}\left(\begin{array}{c}
n \\
n-k
\end{array}\right)(-1)^{n-k}(\alpha)_{n-k} x^{k} \phi^{(k)}(x) .
\end{gathered}
$$


Applying the formula 1.5 to $p(x)=\phi(x)$ and $q(x)=x^{\alpha}$ for $\alpha \in \mathbb{R}$ yields

$$
\begin{aligned}
& \frac{\mathrm{d}^{n} h_{\alpha}(x)}{\mathrm{d} x^{n}}=\frac{(-1)^{n}}{x^{\alpha(n+1)}} \mid \begin{array}{ccc}
\phi(x) & \left(\begin{array}{l}
0 \\
0
\end{array}\right)\langle\alpha\rangle_{0} x^{\alpha} & 0 \\
\phi^{\prime}(x) & \left(\begin{array}{l}
1 \\
0
\end{array}\right)\langle\alpha\rangle_{1} x^{\alpha-1} & \left(\begin{array}{l}
1 \\
1
\end{array}\right)\langle\alpha\rangle_{0} x^{\alpha} \\
\phi^{\prime \prime}(x) & \left(\begin{array}{l}
2 \\
0
\end{array}\right)\langle\alpha\rangle_{2} x^{\alpha-2} & \left(\begin{array}{l}
2 \\
1
\end{array}\right)\langle\alpha\rangle_{1} x^{\alpha-1} \\
\phi^{(3)}(x) & \left(\begin{array}{l}
3 \\
0
\end{array}\right)\langle\alpha\rangle_{3} x^{\alpha-3} & \left(\begin{array}{l}
3 \\
1
\end{array}\right)\langle\alpha\rangle_{2} x^{\alpha-2} \\
\vdots & \vdots & \vdots \\
\phi^{(n-2)}(x) & \left(\begin{array}{c}
n-2 \\
0
\end{array}\right)\langle\alpha\rangle_{n-2} x^{\alpha-(n-2)} & \left(\begin{array}{c}
n-2 \\
\phi^{(n-1)}(x)
\end{array}\right)\langle\alpha\rangle_{n-3} x^{\alpha-(n-3)} \\
\left.\phi^{n-1}\right)\langle\alpha\rangle_{n-1} x^{\alpha-(n-1)} & \left(\begin{array}{c}
n-1 \\
1
\end{array}\right)\langle\alpha\rangle_{n-2} x^{\alpha-(n-2)} \\
\phi^{(n)}(x) & \left(\begin{array}{l}
n \\
0
\end{array}\right)\langle\alpha\rangle_{n} x^{\alpha-n} & \left(\begin{array}{l}
n \\
1
\end{array}\right)\langle\alpha\rangle_{n-1} x^{\alpha-(n-1)}
\end{array}
\end{aligned}
$$

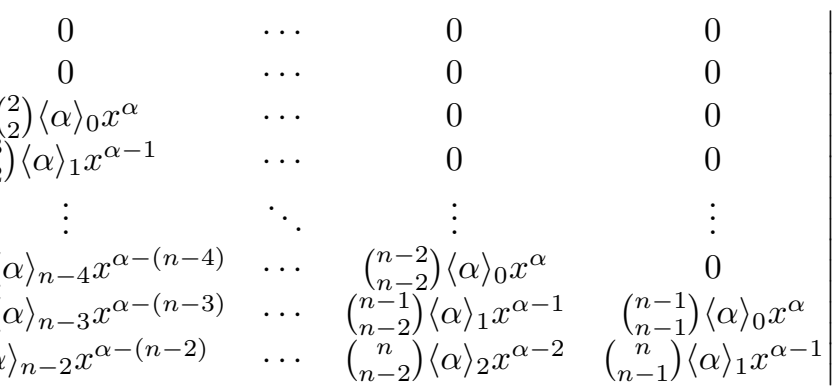

$$
\begin{aligned}
& =\frac{(-1)^{n}}{x^{\alpha(n+1)} x^{n(n+1) / 2}} \begin{array}{ccc}
x^{0} \phi(x) & \left(\begin{array}{l}
0 \\
0
\end{array}\right)\langle\alpha\rangle_{0} x^{\alpha} & 0 \\
x^{1} \phi^{\prime}(x) & \left(\begin{array}{l}
1 \\
0
\end{array}\right)\langle\alpha\rangle_{1} x^{\alpha} & \left(\begin{array}{l}
1 \\
1
\end{array}\right)\langle\alpha\rangle_{0} x^{\alpha+1} \\
x^{2} \phi^{\prime \prime}(x) & \left(\begin{array}{l}
2 \\
0
\end{array}\right)\langle\alpha\rangle_{2} x^{\alpha} & \left(\begin{array}{l}
2 \\
1
\end{array}\right)\langle\alpha\rangle_{1} x^{\alpha+1} \\
x^{3} \phi^{(3)}(x) & \left(\begin{array}{l}
3 \\
0
\end{array}\right)\langle\alpha\rangle_{3} x^{\alpha} & \left(\begin{array}{l}
3 \\
3 \\
1
\end{array}\right)\langle\alpha\rangle_{2} x^{\alpha+1} \\
\vdots & \vdots & \vdots \\
x^{n-2} \phi^{(n-2)}(x) & \left(\begin{array}{c}
n-2 \\
0
\end{array}\right)\langle\alpha\rangle_{n-2} x^{\alpha} & \left(\begin{array}{c}
n-2 \\
1
\end{array}\right)\langle\alpha\rangle_{n-3} x^{\alpha+1} \\
x^{n-1} \phi^{(n-1)}(x) & \left(\begin{array}{c}
n-1 \\
0
\end{array}\right)\langle\alpha\rangle_{n-1} x^{\alpha} & \left(\begin{array}{c}
n-1 \\
1
\end{array}\right)\langle\alpha\rangle_{n-2} x^{\alpha+1} \\
x^{n} \phi^{(n)}(x) & \left(\begin{array}{l}
n \\
0
\end{array}\right)\langle\alpha\rangle_{n} x^{\alpha} & \left(\begin{array}{l}
n \\
1 \\
1
\end{array}\right)\langle\alpha\rangle_{n-1} x^{\alpha+1}
\end{array}
\end{aligned}
$$

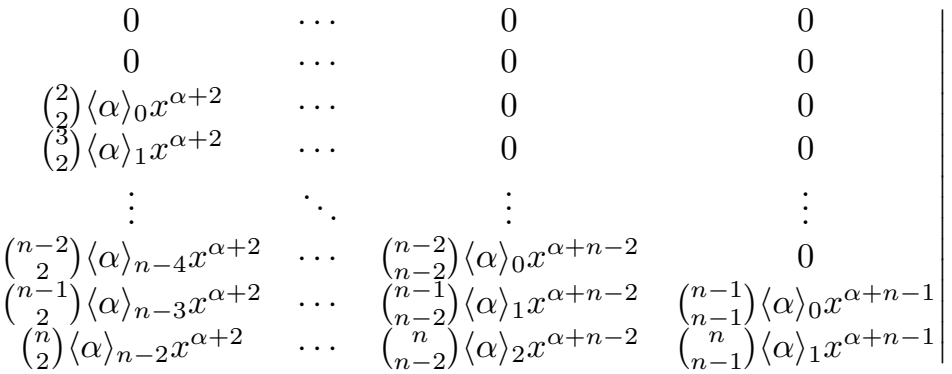

$$
\begin{aligned}
& =\frac{(-1)^{n} x^{(2 \alpha+n-1) n / 2}}{x^{\alpha(n+1)} x^{n(n+1) / 2}} \mid \begin{array}{ccc}
x^{0} \phi(x) & \left(\begin{array}{l}
0 \\
0
\end{array}\right)\langle\alpha\rangle_{0} & 0 \\
x^{1} \phi^{\prime}(x) & \left(\begin{array}{l}
1 \\
0
\end{array}\right)\langle\alpha\rangle_{1} & \left(\begin{array}{l}
1 \\
1
\end{array}\right)\langle\alpha\rangle_{0} \\
x^{2} \phi^{\prime \prime}(x) & \left(\begin{array}{l}
2 \\
0
\end{array}\right)\langle\alpha\rangle_{2} & \left(\begin{array}{l}
2 \\
1
\end{array}\right)\langle\alpha\rangle_{1} \\
x^{3} \phi^{(3)}(x) & \left(\begin{array}{l}
3 \\
0
\end{array}\right)\langle\alpha\rangle_{3} & \left(\begin{array}{l}
3 \\
1
\end{array}\right)\langle\alpha\rangle_{2} \\
\vdots & \vdots & \vdots \\
x^{n-2} \phi^{(n-2)}(x) & \left(\begin{array}{c}
n-2 \\
0
\end{array}\right)\langle\alpha\rangle_{n-2} & \left(\begin{array}{c}
n-2 \\
1
\end{array}\right)\langle\alpha\rangle_{n-3} \\
x^{n-1} \phi^{(n-1)}(x) & \left(\begin{array}{c}
n-1 \\
0
\end{array}\right)\langle\alpha\rangle_{n-1} & \left(\begin{array}{c}
n-1 \\
1
\end{array}\right)\langle\alpha\rangle_{n-2} \\
x^{n} \phi^{(n)}(x) & \left(\begin{array}{c}
n \\
0
\end{array}\right)\langle\alpha\rangle_{n} & \left(\begin{array}{c}
n \\
1 \\
1
\end{array}\right)\langle\alpha\rangle_{n-1}
\end{array}
\end{aligned}
$$


COMPUTATION OF SEVERAL HESSENBERG DETERMINANTS

$$
\begin{aligned}
& \begin{array}{lllll}
0 & \cdots & 0 & 0
\end{array} \\
& \begin{array}{llll}
0 & \cdots & 0 & 0
\end{array} \\
& \left(\begin{array}{l}
2 \\
2
\end{array}\right)\langle\alpha\rangle_{0} \quad \cdots \quad 0 \quad 0 \quad 0 \\
& \left(\begin{array}{c}
3 \\
2
\end{array}\right)\langle\alpha\rangle_{1} \quad \cdots \quad 0 \quad 0 \quad 0
\end{aligned}
$$

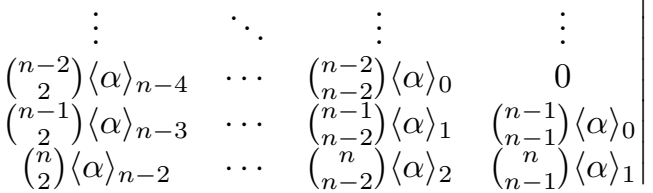

$$
\begin{aligned}
& =\frac{(-1)^{n}}{x^{\alpha+n}} \sum_{k=0}^{n}(-1)^{k+2} x^{k} \phi^{(k)}(x)\left|\begin{array}{cc}
A_{k, k} & O_{k, n-k} \\
C_{n-k, k} & B_{n-k, n-k}
\end{array}\right| \text {, }
\end{aligned}
$$

where $O_{k, n-k}$ is a $k \times(n-k)$ matrix whose entries are all zero,

$$
\begin{aligned}
& A_{k, k}=\left(\left(\begin{array}{l}
i-1 \\
j-1
\end{array}\right)\langle\alpha\rangle_{i-j}\right)_{1 \leq i, j \leq k}
\end{aligned}
$$

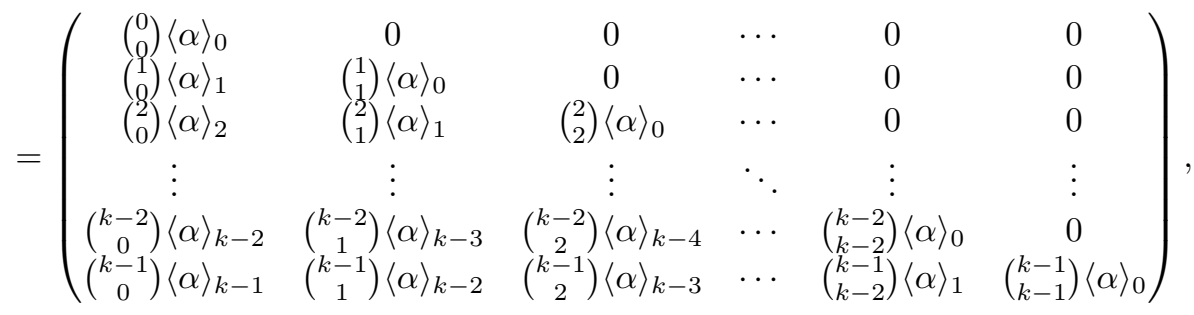

$$
\begin{aligned}
& B_{n-k, n-k}=\left(\left(\begin{array}{l}
i-1 \\
j-1
\end{array}\right)\langle\alpha\rangle_{i-j}\right)_{k+2 \leq i \leq n+1, k+1 \leq j \leq n}
\end{aligned}
$$

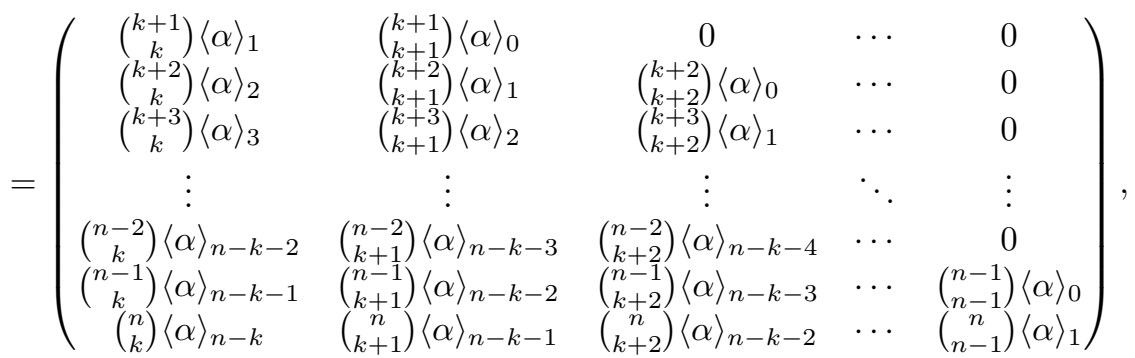

$$
\begin{aligned}
& C_{n-k, k}=\left(\left(\begin{array}{c}
i-1 \\
j-1
\end{array}\right)\langle\alpha\rangle_{i-j}\right)_{k+2 \leq i \leq n+1,1 \leq j \leq k} \\
& =\left(\begin{array}{ccccc}
\left(\begin{array}{c}
k+1 \\
0
\end{array}\right)\langle\alpha\rangle_{k+1} & \left(\begin{array}{c}
k+1 \\
1
\end{array}\right)\langle\alpha\rangle_{k} & \cdots & \left(\begin{array}{c}
k+1 \\
k-2
\end{array}\right)\langle\alpha\rangle_{3} & \left(\begin{array}{c}
k+1 \\
k-1
\end{array}\right)\langle\alpha\rangle_{2} \\
\left(\begin{array}{c}
k+2 \\
0
\end{array}\right)\langle\alpha\rangle_{k+2} & \left(\begin{array}{c}
k+2 \\
1
\end{array}\right)\langle\alpha\rangle_{k+1} & \cdots & \left(\begin{array}{c}
k+2 \\
k-2
\end{array}\right)\langle\alpha\rangle_{4} & \left(\begin{array}{c}
k+2 \\
k-1
\end{array}\right)\langle\alpha\rangle_{3} \\
\vdots & \vdots & \ddots & \vdots & \vdots \\
\left(\begin{array}{c}
n-1 \\
0
\end{array}\right)\langle\alpha\rangle_{n-1} & \left(\begin{array}{c}
n-1 \\
(
\end{array}\right)\langle\alpha\rangle_{n-2} & \cdots & \left(\begin{array}{c}
n-1 \\
k-2
\end{array}\right)\langle\alpha\rangle_{n-k+1} & \left(\begin{array}{c}
n-1 \\
k-1
\end{array}\right)\langle\alpha\rangle_{n-k} \\
\left(\begin{array}{c}
n \\
0
\end{array}\right)\langle\alpha\rangle_{n} & \left(\begin{array}{c}
n \\
1
\end{array}\right)\langle\alpha\rangle_{n-1} & \cdots & (\alpha)\rangle_{n-k+2} & \left(\begin{array}{c}
n \\
k-1
\end{array}\right)\langle\alpha\rangle_{n-k+1}
\end{array}\right) .
\end{aligned}
$$

Employing the formula

$$
\left|\begin{array}{cc}
A & 0 \\
C & B
\end{array}\right|=|A||B|
$$


for square matrices $A$ and $B$, see [2, p. 103, eq. (2.7.6)], we obtain

$$
\begin{aligned}
\frac{\mathrm{d}^{n} h_{\alpha}(x)}{\mathrm{d} x^{n}} & =\frac{(-1)^{n}}{x^{\alpha+n}} \sum_{k=0}^{n}(-1)^{k} x^{k} \phi^{(k)}(x)\left|\begin{array}{cc}
A_{k, k} & O_{k, n-k} \\
C_{n-k, k} & B_{n-k, n-k}
\end{array}\right| \\
& =\frac{(-1)^{n}}{x^{\alpha+n}} \sum_{k=0}^{n}(-1)^{k}\left|A_{k, k}\right|\left|B_{n-k, n-k}\right| x^{k} \phi^{(k)}(x) \\
& =\frac{(-1)^{n}}{x^{\alpha+n}} \sum_{k=0}^{n}(-1)^{k}\left|B_{n-k, n-k}\right| x^{k} \phi^{(k)}(x),
\end{aligned}
$$

where the determinant of zero order is regarded as 1.

Comparing (2.2) and (2.3) and equating the coefficients of $x^{k} \phi^{(k)}$ yield

$$
\left|B_{n-k, n-k}\right|=\left(\begin{array}{c}
n \\
n-k
\end{array}\right)(\alpha)_{n-k}, \quad 0 \leq k \leq n .
$$

By replacing $n-k$ by $\ell$, this equality can be concretely rewritten as

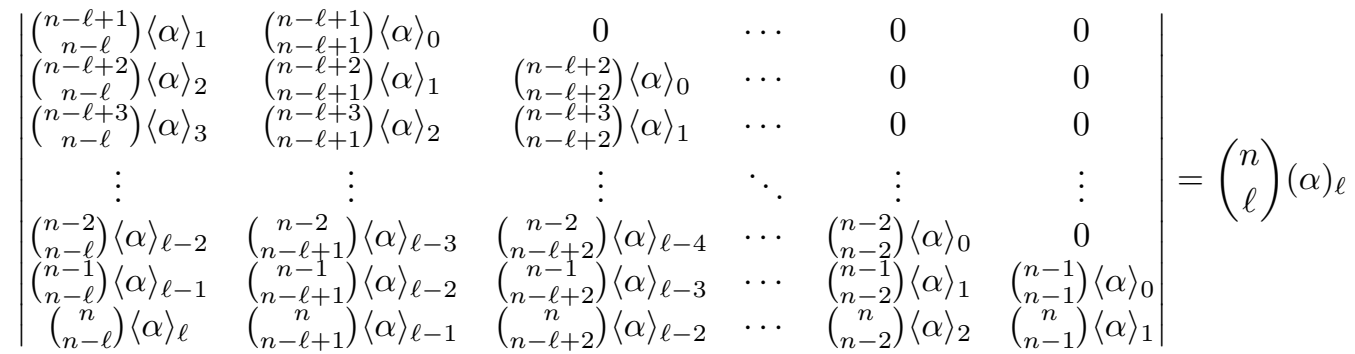

for $\alpha \in \mathbb{R}$ and $0 \leq \ell \leq n$. By the formula $\left(\begin{array}{l}n \\ k\end{array}\right)=\left(\begin{array}{c}n \\ n-k\end{array}\right)$, we can write 2.4 as

$$
\begin{aligned}
& \mid \begin{array}{cccc}
\left(\begin{array}{c}
n-\ell+1 \\
1
\end{array}\right)\langle\alpha\rangle_{1} & \left(\begin{array}{c}
n-\ell+1 \\
0
\end{array}\right)\langle\alpha\rangle_{0} & 0 & \cdots \\
\left(\begin{array}{l}
n-\ell+2 \\
2
\end{array}\right)\langle\alpha\rangle_{2} & \left(\begin{array}{c}
n-\ell+2 \\
1
\end{array}\right)\langle\alpha\rangle_{1} & \left(\begin{array}{c}
n-\ell+2 \\
0
\end{array}\right)\langle\alpha\rangle_{0} & \ldots \\
\left(\begin{array}{c}
n-\ell+3 \\
3
\end{array}\right)\langle\alpha\rangle_{3} & \left(\begin{array}{c}
n-\ell+3 \\
2
\end{array}\right)\langle\alpha\rangle_{2} & \left(\begin{array}{c}
n-\ell+3 \\
1
\end{array}\right)\langle\alpha\rangle_{1} & \ldots \\
\vdots & \vdots & \vdots & \ddots \\
\left(\begin{array}{c}
n-\ell+(\ell-2) \\
\ell-2
\end{array}\right)\langle\alpha\rangle_{\ell-2} & \left(\begin{array}{c}
n-\ell+(\ell-2) \\
\ell-3
\end{array}\right)\langle\alpha\rangle_{\ell-3} & \left(\begin{array}{c}
n-\ell+(\ell-2) \\
\ell-4
\end{array}\right)\langle\alpha\rangle_{\ell-4} & \cdots \\
\left(\begin{array}{c}
n-\ell+(\ell-1) \\
\ell-1
\end{array}\right)\langle\alpha\rangle_{\ell-1} & \left(\begin{array}{c}
n-\ell+(\ell-1) \\
\ell-2
\end{array}\right)\langle\alpha\rangle_{\ell-2} & \left(\begin{array}{c}
n-\ell+(\ell-1) \\
\ell-3
\end{array}\right)\langle\alpha\rangle_{\ell-3} & \cdots \\
\left(\begin{array}{c}
n-\ell+\ell \\
\ell
\end{array}\right)\langle\alpha\rangle_{\ell} & \left(\begin{array}{c}
n-\ell+\ell \\
\ell-1
\end{array}\right)\langle\alpha\rangle_{\ell-1} & \left(\begin{array}{c}
n-\ell+\ell \\
\ell-2
\end{array}\right)\langle\alpha\rangle_{\ell-2} & \cdots
\end{array} \\
& \begin{array}{llll}
\ldots & 0 & 0 & 0 \\
\ldots & 0 & 0 & 0
\end{array} \\
& \begin{array}{llll}
\ldots & 0 & 0 & 0
\end{array} \\
& \begin{array}{ccc}
\ddots & \vdots & \vdots
\end{array} \\
& \cdots \quad\left(\begin{array}{c}
n-\ell+(\ell-2) \\
1
\end{array}\right)\langle\alpha\rangle_{1} \quad\left(\begin{array}{c}
n-\ell+(\ell-2) \\
0
\end{array}\right)\langle\alpha\rangle_{0} \quad 0 \\
& \ldots \quad\left(\begin{array}{cc}
n-\ell+(\ell-1) \\
2
\end{array}\right)\langle\alpha\rangle_{2} \quad\left(\begin{array}{c}
n-\ell+(\ell-1) \\
1
\end{array}\right)\langle\alpha\rangle_{1} \quad\left(\begin{array}{c}
n-\ell+(\ell-1) \\
0
\end{array}\right)\langle\alpha\rangle_{0}
\end{aligned}
$$

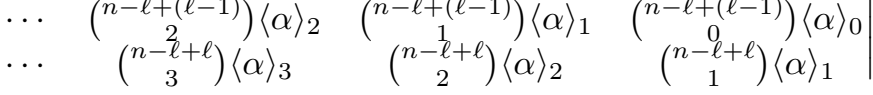

$$
\begin{aligned}
& =\left|\left(\begin{array}{c}
n-\ell+i \\
i-j+1
\end{array}\right)\langle\alpha\rangle_{i-j+1}\right|_{1 \leq i, j \leq \ell}=\left(\begin{array}{c}
n-\ell+\ell \\
\ell
\end{array}\right)(\alpha)_{\ell}
\end{aligned}
$$

for $\alpha \in \mathbb{R}$ and $0 \leq \ell \leq n$. Replacing $n-\ell \geq 0$ by $m \geq 0$ in 2.5 leads to the formula (2.1) for $m \geq 0$ and $\alpha \in \mathbb{R}$. 
Since both sides of the equality 2.1 are analytic with respect to $\alpha \in \mathbb{C}$, by the uniqueness theorem of analytic functions, see [8, p. 90, Theorem 1.2], the formula 2.1 is valid for $\alpha \in \mathbb{C}$. The first proof of Theorem 2.1 is complete.

Second proof. Consider the $\ell \times \ell$ Hessenberg matrix

$$
H_{\ell, \ell}=\left(\begin{array}{cccccc}
t_{1,1} & 1 & 0 & \cdots & 0 & 0 \\
t_{2,1} & t_{2,2} & 1 & \cdots & 0 & 0 \\
t_{3,1} & t_{3,2} & t_{3,3} & \cdots & 0 & 0 \\
\vdots & \vdots & \vdots & \ddots & \vdots & \vdots \\
t_{\ell-2,1} & t_{\ell-2,2} & t_{\ell-2,3} & \cdots & 1 & 0 \\
t_{\ell-1,1} & t_{\ell-1,2} & t_{\ell-1,3} & \cdots & t_{\ell-1, \ell-1} & 1 \\
t_{\ell, 1} & t_{\ell, 2} & t_{\ell, 3} & \cdots & t_{\ell, \ell-1} & t_{\ell, \ell}
\end{array}\right) .
$$

The co-matrix of the entry $t_{\ell, 1}$ of $H_{\ell, \ell}$ is a lower triangular matrix whose entries on the diagonal are all 1 , so $\operatorname{cof}\left(t_{\ell, 1}\right)=(-1)^{\ell+1}$. For $j>1$, the co-matrix of the entry $t_{\ell, j}$ of $H_{\ell, \ell}$ has the block structure

$$
\left(\begin{array}{cc}
H_{(j-1) \times(j-1)} & O_{(j-1) \times(\ell-j)} \\
V_{(\ell-j) \times(j-1)} & U_{(\ell-j) \times(\ell-j)}
\end{array}\right),
$$

where $O_{(j-1) \times(\ell-j)}$ is a $(j-1) \times(\ell-j)$ matrix whose elements are all zero, $U_{(\ell-j) \times(\ell-j)}$ is a lower triangular matrix whose entries on the diagonal are all 1 , and $H_{(j-1) \times(j-1)}=\left(t_{p, q}\right)_{1 \leq p, q \leq j-1}$ is the $(j-1) \times(j-1)$ principal sub-matrix of $H_{\ell, \ell}$. Hence,

$$
\operatorname{cof}\left(t_{\ell, j}\right)=(-1)^{\ell+j}\left|H_{(j-1) \times(j-1)}\right| .
$$

As a result, we obtain the recurrence formula

$$
\left|H_{\ell, \ell}\right|=(-1)^{\ell+1} t_{\ell, 1}+\sum_{j=2}^{\ell}(-1)^{\ell+j} t_{\ell, j}\left|H_{(j-1) \times(j-1)}\right| .
$$

For $m \in\{0\} \cup \mathbb{N}$ and $\alpha \in \mathbb{C}$, set $t_{i, j}=\left(\begin{array}{c}m+i \\ i-j+1\end{array}\right)\langle\alpha\rangle_{i-j+1}$ for $1 \leq i, j \leq \ell$ and define $H(m, \ell, \alpha)=$ $\left|\left(\begin{array}{c}m+i \\ i-j+1\end{array}\right)\langle\alpha\rangle_{i-j+1}\right|_{1 \leq i, j \leq \ell}$. We will prove by induction on $\ell$ that $H(m, \ell, \alpha)=\left(\begin{array}{c}m+\ell \\ \ell\end{array}\right)(\alpha)_{\ell}$. Indeed, applying 2.6. to the determinant $H(m, \ell, \alpha)$ immediately results in

$$
\begin{aligned}
H(m, \ell, \alpha) & =(-1)^{\ell+1}\left(\begin{array}{c}
m+\ell \\
\ell
\end{array}\right)\langle\alpha\rangle_{\ell}+\sum_{j=2}^{\ell}(-1)^{\ell+j}\left(\begin{array}{c}
m+\ell \\
\ell-j+1
\end{array}\right)\langle\alpha\rangle_{\ell-j+1} H(m, j-1, \alpha) \\
& =(-1)^{\ell+1}\left(\begin{array}{c}
m+\ell \\
\ell
\end{array}\right)\langle\alpha\rangle_{\ell}+\sum_{j=2}^{\ell}(-1)^{\ell+j}\left(\begin{array}{c}
m+\ell \\
\ell-j+1
\end{array}\right)\langle\alpha\rangle_{\ell-j+1}\left(\begin{array}{c}
m+j-1 \\
j-1
\end{array}\right)(\alpha)_{j-1} \\
& =\sum_{j=1}^{\ell}(-1)^{\ell+j}\left(\begin{array}{c}
m+\ell \\
\ell-j+1
\end{array}\right)\langle\alpha\rangle_{\ell-j+1}\left(\begin{array}{c}
m+j-1 \\
j-1
\end{array}\right)(\alpha)_{j-1} \\
& =\left(\begin{array}{c}
m+\ell \\
\ell
\end{array}\right) \sum_{j=1}^{\ell}(-1)^{\ell+j}\left(\begin{array}{c}
\ell \\
j-1
\end{array}\right)\langle\alpha\rangle_{\ell-j+1}(\alpha)_{j-1} \\
& =\left(\begin{array}{c}
m+\ell \\
\ell
\end{array}\right) \sum_{j=0}^{\ell-1}(-1)^{\ell+j+1}\left(\begin{array}{l}
\ell \\
j
\end{array}\right)\langle\alpha\rangle_{\ell-j}(\alpha)_{j} \\
& =\left(\begin{array}{c}
m+\ell \\
\ell
\end{array}\right) \sum_{j=0}^{\ell-1}(-1)^{\ell+j+1}\left(\begin{array}{l}
\ell \\
j
\end{array}\right)\langle\alpha\rangle_{\ell-j}(\alpha)_{j}
\end{aligned}
$$




$$
\begin{aligned}
& =\alpha\left(\begin{array}{c}
m+\ell \\
\ell
\end{array}\right) \sum_{j=0}^{\ell-1}(-1)^{\ell-j+1}\left(\begin{array}{l}
\ell \\
j
\end{array}\right)(\alpha+j+1-\ell)_{\ell-1} \\
& =\alpha\left(\begin{array}{c}
m+\ell \\
\ell
\end{array}\right) \sum_{j=1}^{\ell}(-1)^{j+1}\left(\begin{array}{l}
\ell \\
j
\end{array}\right)(\alpha+1-j)_{\ell-1},
\end{aligned}
$$

where we used the facts $\left(\begin{array}{c}m+\ell \\ \ell-j+1\end{array}\right)\left(\begin{array}{c}m+j-1 \\ j-1\end{array}\right)=\left(\begin{array}{c}m+\ell \\ \ell\end{array}\right)\left(\begin{array}{c}\ell \\ j-1\end{array}\right)$ and $\langle\alpha\rangle_{\ell-j}(\alpha)_{j}=\alpha(\alpha+j+1-\ell)_{\ell-1}$. Since $(\alpha+1-x)_{\ell-1}$ is a polynomial of $x$ and of degree $\ell-1$, by virtue of [40, p. 68, Eq. (6.16)], its $\ell$ th finite difference is 0 , that is,

$$
\sum_{j=0}^{\ell}(-1)^{j}\left(\begin{array}{l}
\ell \\
j
\end{array}\right)(\alpha+1-j)_{\ell-1}=0
$$

which is equivalent to

$$
\sum_{j=1}^{\ell}(-1)^{j+1}\left(\begin{array}{l}
\ell \\
j
\end{array}\right)(\alpha+1-j)_{\ell-1}=(\alpha+1)_{\ell-1} .
$$

Consequently, we have

$$
H(m, \ell, \alpha)=\left(\begin{array}{c}
m+\ell \\
\ell
\end{array}\right) \alpha(\alpha+1)_{\ell-1}=\left(\begin{array}{c}
m+\ell \\
\ell
\end{array}\right)(\alpha)_{\ell} .
$$

The second proof of Theorem 2.1 is complete.

Remark 2.1. On 8 August 2019, Zivorad Tomovski (Ss. Cyril and Methodius University, Macedonia) mentioned on ResearchGate that one idea for the proof of Theorem 2.1 appeared in [42.

We guess that the formula (2.1) in Theorem 2.1 should be valid for $m \in \mathbb{C}$. In fact, both sides of (2.1) are polynomials in $m$ and, since they are equal for infinitely many $m$ 's, they are equal as polynomials, so we may replace $m$ by any complex number $z \in \mathbb{C}$. We now confirm this guess by supplying a direct proof.

Theorem 2.2. For $z, \alpha \in \mathbb{C}$ and $\ell \in \mathbb{N}$, we have

$$
\begin{aligned}
& \left|\begin{array}{cccccc|}
\left(\begin{array}{c}
z+1 \\
1
\end{array}\right)\langle\alpha\rangle_{1} & \left(\begin{array}{c}
z+1 \\
0
\end{array}\right)\langle\alpha\rangle_{0} & 0 & \cdots & 0 & 0 \\
\left(\begin{array}{c}
z+2 \\
2
\end{array}\right)\langle\alpha\rangle_{2} & \left(\begin{array}{c}
z+2 \\
1
\end{array}\right)\langle\alpha\rangle_{1} & \left(\begin{array}{c}
z+2 \\
0
\end{array}\right)\langle\alpha\rangle_{0} & \cdots & 0 & 0 \\
\left(\begin{array}{c}
z+3 \\
3
\end{array}\right)\langle\alpha\rangle_{3} & \left(\begin{array}{c}
z+3 \\
2
\end{array}\right)\langle\alpha\rangle_{2} & \left(\begin{array}{c}
z+3 \\
1
\end{array}\right)\langle\alpha\rangle_{1} & \cdots & 0 & 0 \\
\vdots & \vdots & \vdots & \ddots & \vdots & \vdots \\
\left(\begin{array}{c}
z+\ell-2 \\
\ell-2
\end{array}\right)\langle\alpha\rangle_{\ell-2} & \left(\begin{array}{c}
z+\ell-2 \\
\ell-3
\end{array}\right)\langle\alpha\rangle_{\ell-3} & \left(\begin{array}{c}
z+\ell-2 \\
\ell-4
\end{array}\right)\langle\alpha\rangle_{\ell-4} & \cdots & \left(\begin{array}{c}
z+\ell-2 \\
0
\end{array}\right)\langle\alpha\rangle_{0} & 0 \\
\left(\begin{array}{l}
z+\ell-1 \\
\ell-1
\end{array}\right)\langle\alpha\rangle_{\ell-1} & \left(\begin{array}{c}
z+\ell-1 \\
\ell-2
\end{array}\right)\langle\alpha\rangle_{\ell-2} & \left(\begin{array}{c}
z+\ell-1 \\
\ell-3
\end{array}\right)\langle\alpha\rangle_{\ell-3} & \cdots & \left(\begin{array}{c}
z+\ell-1 \\
1
\end{array}\right)\langle\alpha\rangle_{1} & \left(\begin{array}{c}
z+\ell-1 \\
0
\end{array}\right)\langle\alpha\rangle_{0} \\
\left(\begin{array}{c}
z+\ell \\
\ell
\end{array}\right)\langle\alpha\rangle_{\ell} & \left(\begin{array}{c}
z+\ell \\
\ell-1
\end{array}\right)\langle\alpha\rangle_{\ell-1} & \left(\begin{array}{c}
z+\ell \\
\ell-2
\end{array}\right)\langle\alpha\rangle_{\ell-2} & \cdots & \left(\begin{array}{c}
z+\ell \\
2
\end{array}\right)\langle\alpha\rangle_{2} & \left(\begin{array}{c}
z+\ell \\
1
\end{array}\right)\langle\alpha\rangle_{1}
\end{array}\right| \\
& =\left|\left(\begin{array}{c}
z+i \\
i-j+1
\end{array}\right)\langle\alpha\rangle_{i-j+1}\right|_{1 \leq i, j \leq \ell}=\left(\begin{array}{c}
z+\ell \\
\ell
\end{array}\right)(\alpha)_{\ell},
\end{aligned}
$$

where $\left(\begin{array}{l}z \\ k\end{array}\right)=\frac{\langle z\rangle_{k}}{k !}$ is defined for $z \in \mathbb{C}$ and $k \in\{0\} \cup \mathbb{N}$.

Proof. Let

$$
A_{\ell}=\left(A_{i j}\right)_{1 \leq i, j \leq \ell}=\left|\left(\begin{array}{c}
z+i \\
i-j+1
\end{array}\right)\langle\alpha\rangle_{i-j+1}\right|_{1 \leq i, j \leq \ell}, \quad B_{i j}=\left(\begin{array}{c}
\alpha \\
i-j+1
\end{array}\right), \quad B_{\ell}=\left(B_{i j}\right)_{1 \leq i, j \leq \ell} .
$$

Then straightforward computation shows that $A_{i j}=\frac{(z)_{i+1}}{(z)_{j}} B_{i j}$ and

$$
\left|A_{\ell}\right|=\frac{(z)_{2}(z)_{3} \cdots(z)_{\ell+1}}{(z)_{1}(z)_{2} \cdots(z)_{\ell}}\left|B_{\ell}\right|=\frac{(z)_{\ell+1}}{(z)_{1}}\left|B_{\ell}\right|=\left(\begin{array}{c}
z+\ell \\
\ell
\end{array}\right) \ell !\left|B_{\ell}\right| .
$$


Let

$$
D_{\ell}=D\left(a_{1}, \ldots, a_{\ell}\right)=\left|\begin{array}{ccccccc}
a_{1} & 1 & 0 & \cdots & 0 & 0 & 0 \\
a_{2} & a_{1} & 1 & \cdots & 0 & 0 & 0 \\
a_{3} & a_{2} & a_{1} & \cdots & 0 & 0 & 0 \\
\vdots & \vdots & \vdots & \ddots & \vdots & \vdots & \vdots \\
a_{\ell-2} & a_{\ell-3} & a_{\ell-4} & \cdots & a_{1} & 1 & 0 \\
a_{\ell-1} & a_{\ell-2} & a_{\ell-3} & \cdots & a_{2} & a_{1} & 1 \\
a_{\ell} & a_{\ell-1} & a_{\ell-2} & \cdots & a_{3} & a_{2} & a_{1}
\end{array}\right| .
$$

Expanding this determinant with respect to the first row gives

$$
D_{\ell}=a_{1} D_{\ell-1}-\left|\begin{array}{ccccccc}
a_{2} & 1 & 0 & \cdots & 0 & 0 & 0 \\
a_{3} & a_{1} & 1 & \cdots & 0 & 0 & 0 \\
\vdots & \vdots & \vdots & \ddots & \vdots & \vdots & \vdots \\
a_{\ell-2} & a_{\ell-4} & a_{\ell-5} & \cdots & a_{1} & 1 & 0 \\
a_{\ell-1} & a_{\ell-3} & a_{\ell-4} & \cdots & a_{2} & a_{1} & 1 \\
a_{\ell} & a_{\ell-2} & a_{\ell-3} & \cdots & a_{3} & a_{2} & a_{1}
\end{array}\right| .
$$

Consecutively iterating this procedure shows that

$$
D_{\ell}=\sum_{k=1}^{\ell-3}(-1)^{k-1} a_{k} D_{\ell-k}+(-1)^{\ell-3}\left|\begin{array}{ccc}
a_{\ell-2} & 1 & 0 \\
a_{\ell-1} & a_{1} & 1 \\
a_{\ell} & a_{2} & a_{1}
\end{array}\right| .
$$

Accordingly, we obtain

$$
D_{\ell}=\sum_{k=1}^{\ell-2}(-1)^{k-1} a_{k} D_{\ell-k}+(-1)^{\ell-2} a_{\ell-1} a_{1}+(-1)^{\ell-1} a_{\ell} .
$$

Setting $a_{0}=1, D_{0}=1$, and $D_{1}=a_{1}$. Then the above formula can be written as

$$
\sum_{k=0}^{\ell}(-1)^{k} a_{k} D_{\ell-k}=0, \quad \ell>0 .
$$

Applying the equation (2.9) to the special sequence $a_{k}=\left(\begin{array}{l}\alpha \\ k\end{array}\right)$ for $k \geq 0$ reveals that, when assuming $\left|B_{0}\right|=1$ and $\left|B_{1}\right|=\alpha$, we can derive $D_{k}=\left|B_{k}\right|$ for $k \geq 0$ and

$$
\sum_{k=0}^{\ell}(-1)^{k}\left(\begin{array}{l}
\alpha \\
k
\end{array}\right)\left|B_{\ell-k}\right|=0, \quad \ell>0 .
$$

Let $G(x)=\sum_{k=0}^{\infty}\left|B_{k}\right| x^{k}$. Then the equality 2.10 implies $(1-x)^{\alpha} G(x)=1$. Thus, it follows that $G(x)=(1-x)^{-\alpha}$. This means that

$$
\left|B_{k}\right|=(-1)^{k}\left(\begin{array}{c}
-\alpha \\
k
\end{array}\right)=\frac{(\alpha)_{k}}{k !} .
$$

See also [14, Lemma 2.4]. Substituting the equality 2.11) into 2.8) arrives at $\left|A_{\ell}\right|=\left(\begin{array}{c}z+\ell \\ \ell\end{array}\right)(\alpha)_{\ell}$. The formula 2.7 is thus proved. The proof of Theorem 2.2 is complete.

\section{Corollaries}

Now we derive some corollaries from the formula (2.7) in Theorem 2.2 
Corollary 3.1. For $z, \alpha \in \mathbb{C}$ and $\ell \in \mathbb{N}$, we have

$$
\begin{gathered}
\begin{array}{cccccc}
\langle z+1\rangle_{1}\left(\begin{array}{c}
\alpha \\
1
\end{array}\right) & \langle z+1\rangle_{0}\left(\begin{array}{c}
\alpha \\
0
\end{array}\right) & 0 & \cdots & 0 & 0 \\
\langle z+2\rangle_{2}\left(\begin{array}{c}
\alpha \\
2
\end{array}\right) & \langle z+2\rangle_{1}\left(\begin{array}{c}
\alpha \\
1
\end{array}\right) & \langle z+2\rangle_{0}\left(\begin{array}{c}
\alpha \\
0
\end{array}\right) & \cdots & 0 & 0 \\
\langle z+3\rangle_{3}\left(\begin{array}{c}
\alpha \\
3
\end{array}\right) & \langle z+3\rangle_{2}\left(\begin{array}{c}
\alpha \\
2
\end{array}\right) & \langle z+3\rangle_{1}\left(\begin{array}{c}
\alpha \\
1
\end{array}\right) & \cdots & 0 & 0 \\
\langle z+4\rangle_{4}\left(\begin{array}{c}
\alpha \\
4
\end{array}\right) & \langle z+4\rangle_{3}\left(\begin{array}{c}
\alpha \\
3
\end{array}\right) & \langle z+4\rangle_{2}\left(\begin{array}{c}
\alpha \\
2
\end{array}\right) & \cdots & 0 & 0 \\
\vdots & \vdots & \vdots & \ddots & \vdots & \vdots \\
\langle z+\ell\rangle_{\ell}\left(\begin{array}{c}
\alpha \\
\ell
\end{array}\right) & \langle z+\ell\rangle_{\ell-1}\left(\begin{array}{c}
\alpha \\
\ell-1
\end{array}\right) & \langle z+\ell\rangle_{\ell-2}\left(\begin{array}{c}
\alpha \\
\ell-2
\end{array}\right) & \cdots & \langle z+\ell\rangle_{2}\left(\begin{array}{c}
\alpha \\
2
\end{array}\right) & \langle z+\ell\rangle_{1}\left(\begin{array}{c}
\alpha \\
1
\end{array}\right)
\end{array} \mid \\
=\left|\langle z+i\rangle_{i-j+1}\left(\begin{array}{c}
\alpha \\
i-j+1
\end{array}\right)\right|_{1 \leq i, j \leq \ell}=\left(\begin{array}{c}
z+\ell \\
\ell
\end{array}\right)(\alpha)_{\ell} .
\end{gathered}
$$

Proof. This follows from substituting

$$
\begin{gathered}
\left(\begin{array}{c}
z+i \\
i-j+1
\end{array}\right)\langle\alpha\rangle_{i-j+1}=\frac{\langle z+i\rangle_{i-j+1}}{(i-j+1) !}\langle\alpha\rangle_{i-j+1} \\
=\langle z+i\rangle_{i-j+1} \frac{\langle\alpha\rangle_{i-j+1}}{(i-j+1) !}=\langle z+i\rangle_{i-j+1}\left(\begin{array}{c}
\alpha \\
i-j+1
\end{array}\right)
\end{gathered}
$$

into the formula (2.7) in Theorem 2.2. The proof of Corollary 3.1 is complete.

Corollary 3.2. For $\alpha \in \mathbb{C}$ and $\ell \in \mathbb{N}$, we have

$$
\begin{gathered}
\qquad \begin{array}{ccccccc}
\langle\alpha\rangle_{1} & 1 & 0 & \cdots & 0 & 0 & 0 \\
\langle\alpha\rangle_{2} & \left(\begin{array}{l}
2 \\
1
\end{array}\right)\langle\alpha\rangle_{1} & 1 & \cdots & 0 & 0 & 0 \\
\langle\alpha\rangle_{3} & \left(\begin{array}{l}
3 \\
1
\end{array}\right)\langle\alpha\rangle_{2} & \left(\begin{array}{l}
3 \\
1
\end{array}\right)\langle\alpha\rangle_{1} & \cdots & 0 & 0 & 0 \\
\vdots & \vdots & \vdots & \ddots & \vdots & \vdots & \vdots \\
\langle\alpha\rangle_{\ell-2} & \left(\begin{array}{c}
\ell-2 \\
1
\end{array}\right)\langle\alpha\rangle_{\ell-3} & \left(\begin{array}{c}
\ell-2 \\
2
\end{array}\right)\langle\alpha\rangle_{\ell-4} & \cdots & \left(\begin{array}{c}
\ell-2 \\
1
\end{array}\right)\langle\alpha\rangle_{1} & 1 & 0 \\
\langle\alpha\rangle_{\ell-1} & \left(\begin{array}{c}
\ell-1 \\
1
\end{array}\right)\langle\alpha\rangle_{\ell-2} & \left(\begin{array}{c}
\ell-1 \\
2
\end{array}\right)\langle\alpha\rangle_{\ell-3} & \cdots & \left(\begin{array}{c}
\ell-1 \\
2
\end{array}\right)\langle\alpha\rangle_{2} & \left(\begin{array}{c}
\ell-1 \\
1
\end{array}\right)\langle\alpha\rangle_{1} & 1 \\
\langle\alpha\rangle_{\ell} & \left(\begin{array}{c}
\ell \\
1
\end{array}\right)\langle\alpha\rangle_{\ell-1} & \left(\begin{array}{c}
\ell \\
2
\end{array}\right)\langle\alpha\rangle_{\ell-2} & \cdots & \left(\begin{array}{l}
\ell \\
3
\end{array}\right)\langle\alpha\rangle_{3} & \left(\begin{array}{c}
\ell \\
2
\end{array}\right)\langle\alpha\rangle_{2} & \left(\begin{array}{l}
\ell \\
1
\end{array}\right)\langle\alpha\rangle_{1}
\end{array} \mid \\
=\left|\left(\begin{array}{c}
i \\
i-j+1
\end{array}\right)\langle\alpha\rangle_{i-j+1}\right|_{1 \leq i, j \leq \ell}=(\alpha)_{\ell} .
\end{gathered}
$$

Proof. The formula (3.1) follows from taking $z=0$ in (2.7) of Theorem 2.2 and straightforward simplifying. The proof of Corollary 3.2 is complete.

Corollary 3.3. For $\alpha \in \mathbb{C}$ and $\ell \in \mathbb{N}$, we have

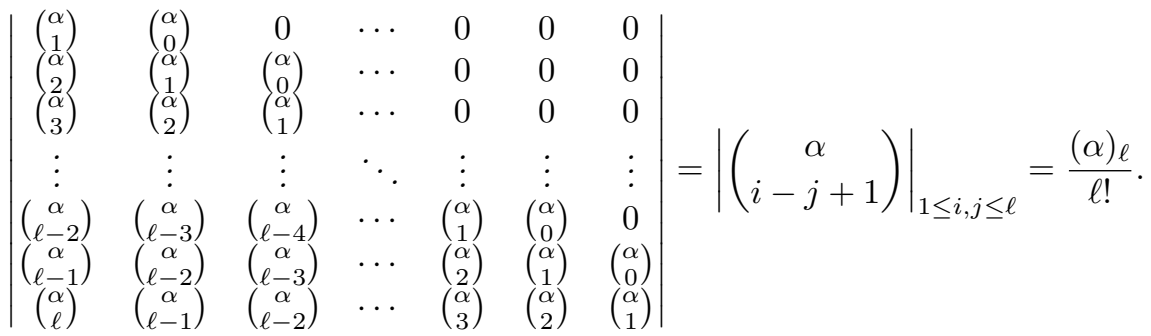

Proof. This follows from substituting

$$
\langle z+i\rangle_{i-j+1}=\frac{(z)_{i+1}}{(z)_{j}}
$$

into Corollary 3.1 and simplifying.

This can also be deduced directly and easily from [14, Lemma 2.4]. 
Lemma 1 in [6] shows that the equality $(3.2)$ holds, because it is equivalent with the relation

$$
\sum_{j=0}^{n}\left(\begin{array}{c}
\alpha \\
n-j
\end{array}\right)\left(\begin{array}{c}
-\alpha \\
j
\end{array}\right)=\left(\begin{array}{l}
0 \\
n
\end{array}\right)
$$

This was observed and announced by Johann Cigler (Universität Wien, Austria) on ResearchGate on 7 August 2019. The proof of Corollary 3.3 is complete.

Corollary 3.4. The formula (2.7) in Theorem 2.2 and the formula (3.2) in Corollary 3.3 are equivalent to each other.

Proof. This follows from reversing those procedures in proofs of Theorem 2.2. Corollary 3.3 , and Corollary 3.1. The proof of Corollary 3.4 is complete.

Corollary 3.5. For $\ell \in \mathbb{N}$, the tridiagonal determinant

$$
\left|\begin{array}{cccccccc}
2 \cdot 1 & 1 & 0 & 0 & \cdots & 0 & 0 & 0 \\
1 \cdot 2 & 2 \cdot 2 & 1 & 0 & \cdots & 0 & 0 & 0 \\
0 & 2 \cdot 3 & 2 \cdot 3 & 1 & \cdots & 0 & 0 & 0 \\
0 & 0 & 3 \cdot 4 & 2 \cdot 4 & \cdots & 0 & 0 & 0 \\
\vdots & \vdots & \vdots & \vdots & \ddots & \vdots & \vdots & \vdots \\
0 & 0 & 0 & 0 & \cdots & 2(\ell-2) & 1 & 0 \\
0 & 0 & 0 & 0 & \cdots & (\ell-2)(\ell-1) & 2(\ell-1) & 1 \\
0 & 0 & 0 & 0 & \cdots & 0 & (\ell-1) \ell & 2 \ell
\end{array}\right|=(\ell+1) !
$$

Proof. The formula (3.3) can be derived from letting $\alpha=2$ in 3.1 or from taking $z=0$ and $\alpha=2$ in (2.7) of Theorem 2.2. The proof of Corollary 3.5 is complete.

Remark 3.1. The formula (3.3) is a special case of [12, Theorem 4.1] and has something to do with tridiagonal determinants in [13, 28] and the closely related references therein.

\section{Recovery of the identities (1.2), (1.3), And (1.4)}

We now recover the identities $(1.2),(1.3)$, and 1.4$)$. These three identities are main results of the paper [5].

Taking $\alpha=m \in \mathbb{N}$ in 2.2 and directly differentiating result in

$$
\begin{aligned}
\frac{\mathrm{d}^{\ell}\left[x^{m+n} h_{m}^{(n)}(x)\right]}{\mathrm{d} x^{\ell}} & =\frac{\mathrm{d}^{\ell}}{\mathrm{d} x^{\ell}} \sum_{k=0}^{n}\left(\begin{array}{c}
n \\
n-k
\end{array}\right)(-1)^{n-k}(m)_{n-k} x^{k} \phi^{(k)}(x) \\
& =\sum_{k=0}^{n}\left(\begin{array}{c}
n \\
n-k
\end{array}\right)(-1)^{n-k}(m)_{n-k} \sum_{i=0}^{\ell}\left(\begin{array}{l}
\ell \\
i
\end{array}\right)\langle k\rangle_{i} x^{k-i} \phi^{(k+\ell-i)}(x) \\
& =\sum_{i=0}^{\ell}\left(\begin{array}{c}
\ell \\
i
\end{array}\right) \sum_{k=0}^{n}\left(\begin{array}{c}
n \\
n-k
\end{array}\right)(-1)^{n-k}(m)_{n-k}\langle k\rangle_{i} x^{k-i} \phi^{(k+\ell-i)}(x) \\
& \rightarrow \sum_{i=0}^{\ell}\left(\begin{array}{c}
\ell \\
i
\end{array}\right)\left(\begin{array}{c}
n \\
n-i
\end{array}\right)(-1)^{n-i}(m)_{n-i}\langle i\rangle_{i} \phi^{(\ell)}(0), \quad x \rightarrow 0 \\
& =(-1)^{n} \phi^{(\ell)}(0) \sum_{i=0}^{\ell}(-1)^{i} i !\left(\begin{array}{c}
\ell \\
i
\end{array}\right)\left(\begin{array}{c}
n \\
n-i
\end{array}\right)(m)_{n-i} \\
& =(-1)^{n} n ! \phi^{(\ell)}(0) \sum_{i=0}^{\ell}(-1)^{i}\left(\begin{array}{c}
\ell \\
i
\end{array}\right)\left(\begin{array}{c}
m+n-i-1 \\
m-1
\end{array}\right)
\end{aligned}
$$




$$
\begin{aligned}
& =(-1)^{n} n !\left(\begin{array}{c}
n+m-\ell-1 \\
m-\ell-1
\end{array}\right) \phi^{(\ell)}(0) \\
& =n !\left(\begin{array}{c}
\ell-m \\
n
\end{array}\right) \phi^{(\ell)}(0)
\end{aligned}
$$

where the formulas

$$
\sum_{k=0}^{n}(-1)^{k}\left(\begin{array}{l}
n \\
k
\end{array}\right)\left(\begin{array}{c}
x-k \\
r
\end{array}\right)=\left(\begin{array}{l}
x-n \\
r-n
\end{array}\right) \text { and }\left(\begin{array}{l}
n \\
k
\end{array}\right)=\left(\begin{array}{c}
-n+k-1 \\
k
\end{array}\right)(-1)^{k}
$$

in [41, p. 65, Eq. (3.49)] and [41, p. 5] were used in sequence in the last two steps. We can also regard the quantity $\sum_{i=0}^{\ell}(-1)^{i}\left(\begin{array}{c}\ell \\ i\end{array}\right)\left(\begin{array}{c}m+n-i-1 \\ m-1\end{array}\right)$ as the coefficient of $x^{n}$ in the binomial expansion of $(1-x)^{\ell-m}$. Therefore, it follows that

$$
\left.\frac{\mathrm{d}^{\ell}\left[x^{n+m} h_{m}^{(n)}(x)\right]}{\mathrm{d} x^{\ell}}\right|_{x=0}= \begin{cases}n !\left(\begin{array}{c}
\ell-m \\
n
\end{array}\right) \phi^{(\ell)}(0), & \ell \geq n+m ; \\
0, & \ell<n+m .\end{cases}
$$

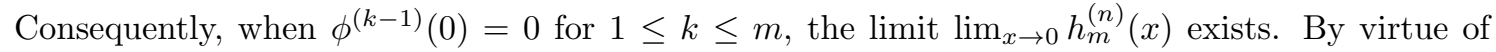
L'Hôspital's rule, we obtain

$$
\begin{gathered}
\lim _{x \rightarrow 0} h_{m}^{(n)}(x)=\lim _{x \rightarrow 0} \frac{x^{n+m} h_{m}^{(n)}(x)}{x^{n+m}}=\lim _{x \rightarrow 0} \frac{\left[x^{n+m} h_{m}^{(n)}(x)\right]^{\prime}}{\left(x^{n+m}\right)^{\prime}} \\
=\lim _{x \rightarrow 0} \frac{\left[x^{n+m} h_{m}^{(n)}(x)\right]^{\prime \prime}}{\left(x^{n+m}\right)^{\prime \prime}}=\cdots=\lim _{x \rightarrow 0} \frac{\left[x^{n+m} h_{m}^{(n)}(x)\right]^{(m+n-1)}}{\left(x^{n+m}\right)^{(m+n-1)}} \\
=\lim _{x \rightarrow 0} \frac{\left[x^{n+m} h_{m}^{(n)}(x)\right]^{(m+n)}}{\left(x^{n+m}\right)^{(m+n)}}=\frac{n !}{(n+m) !} \phi^{(m+n)}(0) .
\end{gathered}
$$

Consequently, the expression for $h_{m}^{(n)}(0)$ in 1.2 is recovered without using the formula

$$
\sum_{q=0}^{n}\left(\begin{array}{l}
n \\
q
\end{array}\right) \frac{(-1)^{q}}{k+q}=\frac{1}{k\left(\begin{array}{c}
k+n \\
k
\end{array}\right)}, \quad k \geq 1, \quad n \geq 0
$$

in [27, Theorem 1] and [40, p. 80, Eq. (7.5)], which was used in [5] to prove the expression for $h_{m}^{(n)}(0)$ in 1.2 .

We observe that the above function $h_{m}(x)$ and its $n$th derivative $h_{m}^{(n)}(x)$ can be represented respectively as integral representations

$$
h_{m}(x)=\frac{1}{(m-1) !} \int_{0}^{1}(1-t)^{m-1} \phi^{(m)}(x t) \mathrm{d} t
$$

and

$$
h_{m}^{(n)}(x)=\frac{1}{(m-1) !} \int_{0}^{1}(1-t)^{m-1} t^{n} \phi^{(m+n)}(x t) \mathrm{d} t .
$$

The equality 1.3 is equivalent to

$$
\frac{\mathrm{d}^{m}\left[x^{n+m} h_{m}^{(n)}(x)\right]}{\mathrm{d} x^{m}}=x^{n} \phi^{(n+m)}(x) .
$$


Utilizing the integral representation (4.1) gives

$$
\begin{aligned}
x^{n+m} h_{m}^{(n)}(x) & =\frac{x^{n+m}}{(m-1) !} \int_{0}^{1}(1-t)^{m-1} t^{n} \phi^{(m+n)}(x t) \mathrm{d} t \\
& =\frac{1}{(m-1) !} \int_{0}^{1} \frac{(1-t)^{m-1}}{t^{m}}(x t)^{m+n} \phi^{(m+n)}(x t) \mathrm{d} t \\
& =\frac{1}{(m-1) !} \int_{0}^{x}(x-u)^{m-1} u^{n} \phi^{(m+n)}(u) \mathrm{d} u
\end{aligned}
$$

Lemma 2.1 in [17 reads that

$$
\frac{\mathrm{d}}{\mathrm{d} x} \int_{x_{0}}^{x} f(u, x) \mathrm{d} u=f(x, x)+\int_{x_{0}}^{x} \frac{\partial f(u, x)}{\partial x} \mathrm{~d} u,
$$

where $f(u, x)$ is differentiable in $x$ and continuous in $(u, x)$ in some region of the $(u, x)$-plane. Accordingly, we obtain

$$
\begin{gathered}
\frac{\mathrm{d}\left[x^{n+m} h_{m}^{(n)}(x)\right]}{\mathrm{d} x}=\frac{1}{(m-1) !} \frac{\mathrm{d}}{\mathrm{d} x} \int_{0}^{x}(x-u)^{m-1} u^{n} \phi^{(m+n)}(u) \mathrm{d} u \\
=\frac{1}{(m-1) !}\left[\left.(x-u)^{m-1} u^{n} \phi^{(m+n)}(u)\right|_{u=x}+\int_{0}^{x} \frac{\mathrm{d}\left[(x-u)^{m-1}\right]}{\mathrm{d} x} u^{n} \phi^{(m+n)}(u) \mathrm{d} u\right] \\
=\frac{1}{(m-2) !} \int_{0}^{x}(x-u)^{m-2} u^{n} \phi^{(m+n)}(u) \mathrm{d} u .
\end{gathered}
$$

Inductively, for $0 \leq \ell<m$, we have

$$
\frac{\mathrm{d}^{\ell}\left[x^{n+m} h_{m}^{(n)}(x)\right]}{\mathrm{d} x^{\ell}}=\frac{1}{(m-1-\ell) !} \int_{0}^{x}(x-u)^{m-1-\ell} u^{n} \phi^{(m+n)}(u) \mathrm{d} u .
$$

In particular,

As a result, we have

$$
\frac{\mathrm{d}^{m-1}\left[x^{n+m} h_{m}^{(n)}(x)\right]}{\mathrm{d} x^{m-1}}=\int_{0}^{x} u^{n} \phi^{(m+n)}(u) \mathrm{d} u .
$$

$$
\frac{\mathrm{d}^{m}\left[x^{n+m} h_{m}^{(n)}(x)\right]}{\mathrm{d} x^{m}}=\frac{\mathrm{d}}{\mathrm{d} x} \int_{0}^{x} u^{n} \phi^{(m+n)}(u) \mathrm{d} u=x^{n} \phi^{(m+n)}(x) .
$$

Consequently, the equality 1.3 is recovered simply.

From the integral representation 4.1), the expression for $h_{m}^{(n)}(0)$ in 1.2 and the conclusions in (1.4) can be recovered immediately.

\section{REFERENCES}

[1] ANDERSON, G.-D.-VAMANAMURTHY, M.-K.-VUORINEN, M.: Conformal Invariants, Inequalities, and Quasiconformal Maps, Canadian Mathematical Society Series of Monographs and Advanced Texts, John Wiley \& Sons, New York, 1997.

[2] BERNSTEIN, D. S.: Scalar, Vector, and Matrix Mathematics: Theory, Facts, and Formulas, Revised and expanded edition, Princeton University Press, Princeton, NJ, 2018.

[3] BOURBAKI, N.: Functions of a Real Variable, Elementary Theory, Translated from the 1976 French original by Philip Spain, Elements of Mathematics (Berlin), Springer-Verlag, Berlin, 2004; available online at https: //doi.org/10.1007/978-3-642-59315-4.

[4] CHEN, C.-P.: Complete monotonicity and logarithmically complete monotonicity properties for the gamma and psi functions, J. Math. Anal. Appl. 336 (2007), 812-822; available online at https://doi.org/10.1016/ j.jmaa.2007.03.028

[5] CHEN, C.-P.-CHOI, J.: Inequalities and complete monotonicity for the gamma and related functions, Commun. Korean Math. Soc. 34 (2019), no. 4, 1261-1278; available online at https://doi.org/10.4134/CKMS. c180450 
F. QI, O. KOUBA, AND I. KADDOURA

[6] CIGLER, J: Some observations about determinants which are connected with Catalan numbers and related topics, arXiv prepint (2019), available online at https://arxiv.org/abs/1902.10468

[7] HARDY, G. H.-LITTLEWOOD, J. E.-PÓLYA, G.: Inequalities, 2nd edition, Cambridge University Press, Cambridge, 1952.

[8] LANG, S.: Complex Analysis, Fourth edition, Graduate Texts in Mathematics, Vol. 103, Springer-Verlag, New York, 1999; available online at https://doi.org/10.1007/978-1-4757-3083-8

[9] QI, F.: A determinantal representation for derangement numbers, Glob. J. Math. Anal. 4 (2016), no. 3, 17-17; available online at https://doi.org/10.14419/gjma.v4i3.6574

[10] QI, F.: Derivatives of tangent function and tangent numbers, Appl. Math. Comput. 268 (2015), 844-858; available online at https://doi.org/10.1016/j.amc.2015.06.123

[11] QI, F.: Determinantal expressions and recurrence relations for Fubini and Eulerian polynomials, J. Interdiscip. Math. 22 (2019), no. 3, 317-335; available online at https://doi.org/10.1080/09720502.2019.1624063

[12] QI, F.-ČERŇANOVÁ, V.-SEMENOV, Y. S.: Some tridiagonal determinants related to central Delannoy numbers, the Chebyshev polynomials, and the Fibonacci polynomials, Politehn. Univ. Bucharest Sci. Bull. Ser. A Appl. Math. Phys. 81 (2019), no. 1, 123-136.

[13] QI, F.-ČERŇANOVÁ, V.-SHI, X.-T.-GUO, B.-N.: Some properties of central Delannoy numbers, J. Comput. Appl. Math. 328 (2018), 101-115; available online at https://doi.org/10.1016/j.cam.2017.07.013.

[14] QI, F.-CHAPMAN, R. J.: Two closed forms for the Bernoulli polynomials, J. Number Theory 159 (2016), 89-100; available online at https://doi.org/10.1016/j.jnt.2015.07.021.

[15] QI, F.-CHEN, S.-X.: Complete monotonicity of the logarithmic mean, Math. Inequal. Appl. 10 (2007), no. 4, 799-804; available online at https://doi.org/10.7153/mia-10-73

[16] QI, F.-CHEN, S.-X.-CHEN, C.-P.: Monotonicity of ratio between the generalized logarithmic means, Math. Inequal. Appl. 10 (2007), no. 3, 559-564; available online at https://doi.org/10.7153/mia-10-52.

[17] QI, F.-DEBNATH, L.: Evaluation of a class of definite integrals, Internat. J. Math. Ed. Sci. Tech. 32 (2001), no. 4, 629-633; available online at http://dx.doi.org/10.1080/00207390116734

[18] QI, F.-GUO, B.-N.: A closed form for the Stirling polynomials in terms of the Stirling numbers, Tbilisi Math. J. 10 (2017), no. 4, 153-158; available online at https://doi.org/10.1515/tmj-2017-0053

[19] QI, F.-GUO, B.-N.: A determinantal expression and a recurrence relation for the Euler polynomials, Adv. Appl. Math. Sci. 16 (2017), no. 9, 297-309.

[20] QI, F.-GUO, B.-N.: A diagonal recurrence relation for the Stirling numbers of the first kind, Appl. Anal. Discrete Math. 12 (2018), no. 1, 153-165; available online at https://doi.org/10.2298/AADM170405004Q.

[21] QI, F.-GUO, B.-N.: Explicit and recursive formulas, integral representations, and properties of the large Schröder numbers, Kragujevac J. Math. 41 (2017), no. 1, 121-141; available online at https://doi.org/10. 5937/KgJMath1701121F

[22] QI, F.-GUO, B.-N.: Expressing the generalized Fibonacci polynomials in terms of a tridiagonal determinant, Matematiche (Catania) 72 (2017), no. 1, 167-175; available online at https://doi.org/10.4418/2017.72.1.13

[23] QI, F.-GUO, B.-N.: Some determinantal expressions and recurrence relations of the Bernoulli polynomials, Mathematics 4 (2016), no. 4, Article 65, 11 pages; available online at https://doi.org/10.3390/math4040065

[24] QI, F.-GUO, B.-N.: Two nice determinantal expressions and a recurrence relation for the Apostol-Bernoulli polynomials, J. Indones. Math. Soc. (MIHMI) 23 (2017), no. 1, 81-87; available online at https://doi.org/ 10.22342/jims.23.1.274.81-87.

[25] QI, F.-KıZıLATEŞ, C.-DU, W.-S.: A closed formula for the Horadam polynomials in terms of a tridiagonal determinant, Symmetry 11 (2019), no. 6, 8 pages; available online at https://doi.org/10.3390/sym11060782

[26] QI, F.-LIM, D.-GUO, B.-N.: Explicit formulas and identities for the Bell polynomials and a sequence of polynomials applied to differential equations, Rev. R. Acad. Cienc. Exactas Fís. Nat. Ser. A Mat. RACSAM 113 (2019), no. 1, 1-9; available online at https://doi.org/10.1007/s13398-017-0427-2

[27] QI, F.-LIM, D.-YAO, Y.-H.: Notes on two kinds of special values for the Bell polynomials of the second kind, Miskolc Math. Notes 20 (2019), no. 1, 465-474; available online at https://doi.org/10.18514/MMN.2019.2635

[28] QI, F.-LIU, A.-Q.: Alternative proofs of some formulas for two tridiagonal determinants, Acta Univ. Sapientiae Math. 10 (2018), no. 2, 287-297; available online at https://doi.org/10.2478/ausm-2018-0022.

[29] QI, F.-MAhmoud, M.-SHI, X.-T.-LIU, F.-F.: Some properties of the Catalan-Qi function related to the Catalan numbers, SpringerPlus 2016, 5:1126, 20 pages; available online at https://doi.org/10.1186/ s40064-016-2793-1.

[30] QI, F.-NIU, D.-W.-GUO, B.-N.: Some identities for a sequence of unnamed polynomials connected with the Bell polynomials, Rev. R. Acad. Cienc. Exactas Fís. Nat. Ser. A Math. RACSAM 113 (2019), no. 2, 557-567; available online at https://doi.org/10.1007/s13398-018-0494-z

[31] QI, F.-NIU, D.-W.-LIM, D.-GUO, B.-N.: Closed formulas and identities for the Bell polynomials and falling factorials, Contrib. Discrete Math. 15 (2020), no. 1, 163-174; available online at https://doi.org/10. $11575 / \mathrm{cdm} . \mathrm{v} 15 \mathrm{i1} .68111$ 
[32] QI, F.-SHI, X.-T.-GUO, B.-N.: Two explicit formulas of the Schröder numbers, Integers 16 (2016), Paper No. A23, 15 pages.

[33] QI, F.-SHI, X.-T.-LIU, F.-F.: Several identities involving the falling and rising factorials and the Cauchy, Lah, and Stirling numbers, Acta Univ. Sapientiae Math. 8 (2016), no. 2, 282-297; available online at https: //doi.org/10.1515/ausm-2016-0019

[34] QI, F.-SHI, X.-T.-LIU, F.-F.-KRUCHININ, D. V.: Several formulas for special values of the Bell polynomials of the second kind and applications, J. Appl. Anal. Comput. 7 (2017), no. 3, 857-871; available online at https://doi.org/10.11948/2017054.

[35] QI, F.-WANG, J.-L.-GUO, B.-N.: A determinantal expression for the Fibonacci polynomials in terms of a tridiagonal determinant, Bull. Iranian Math. Soc. 45 (2019), no. 6, 1821-1829; available online at https: //doi.org/10.1007/s41980-019-00232-4.

[36] QI, F.-WANG, J.-L.-GUO, B.-N.: A recovery of two determinantal representations for derangement numbers, Cogent Math. (2016), 3: 1232878, 7 pages; available online at https://doi.org/10.1080/23311835.2016. 1232878

[37] QI, F.-WANG, J.-L.-GUO, B.-N.: A representation for derangement numbers in terms of a tridiagonal determinant, Kragujevac J. Math. 42 (2018), no. 1, 7-14; available online at https://doi.org/10.5937/ KgJMath1801007F

[38] QI, F.-ZHAO, J.-L.: Some properties of the Bernoulli numbers of the second kind and their generating function, Bull. Korean Math. Soc. 55 (2018), no. 6, 1909-1920; available online at https://doi.org/10.4134/ BKMS.b180039,

[39] QI, F.-ZHAO, J.-L.-GUO, B.-N.: Closed forms for derangement numbers in terms of the Hessenberg determinants, Rev. R. Acad. Cienc. Exactas Fís. Nat. Ser. A Mat. RACSAM 112 (2018), no. 4, 933-944; available online at https://doi.org/10.1007/s13398-017-0401-z.

[40] QUAINTANCE, J.-GOULD, H. W.: Combinatorial Identities for Stirling Numbers, the Unpublished Notes of H. W. Gould, with a foreword by George E. Andrews, World Scientific Publishing Co. Pte. Ltd., Singapore, 2016; available online at https://doi.org/10.1142/9821

[41] SPRUGNOLI, R.: Riordan Array Proofs of Identities in Gould's Book, University of Florence, Italy, 2006.

[42] TOMOVSKI, Ž.-POGÁNY, T. K.-SRIVASTAVA, H. M.: Laplace type integral expressions for a certain three-parameter family of generalized Mittag-Leffler functions with applications involving complete monotonicity, J. Franklin Inst. 351 (2014), no. 12, 5437-5454; available online at https://doi.org/10.1016/j.jfranklin. 2014.09.007

[43] WEI, C.-F.-F. QI: Several closed expressions for the Euler numbers, J. Inequal. Appl. 2015, Paper No. 219, 8 pages; available online at https://doi.org/10.1186/s13660-015-0738-9

${ }^{1}$ Institute of Mathematics, Henan Polytechnic University JiAOZUO 454010, HeNAN

CHINA

2 College of Mathematics, Inner Mongolia University for Nationalities TONGLiaO 028043, InNER MONGOLIA

China

${ }^{3}$ School of Mathematical Sciences, Tianjin Polytechnic University

TIANJIN 300387

CHINA

Email address: qifeng618@gmail.com

$U R L:$ https ://qifeng618. wordpress.com

${ }^{4}$ Department of Mathematics

Higher Institute for Applied Sciences and Technology

P.O.B. 31983, Damascus

SYRIA

Email address: kouba.omran@gmail.com

URL: https://orcid.org/0000-0002-9577-3326

${ }^{5}$ Department of Mathematics

SCHOOL OF ARTS AND SCIENCES

LEBANESE INTERNATIONAL UNIVERSITY

BEIRUT

LEBANON

Email address: issam.kaddoura@liu.edu.lb 